\title{
Constraint-Following Approach for Platoon Control Strategy of Connected Autonomous Vehicles
}

\author{
Mengyan Hu (D), Ying Gao $(\mathbb{D}$, Xiangmo Zhao, Bin Tian (iD, and Zhigang Xu $(\mathbb{D}$ \\ School of Information and Engineering, Chang'an University, Xi'an, Shaanxi, China \\ Correspondence should be addressed to Ying Gao; gaoying888@chd.edu.cn
}

Received 22 November 2021; Revised 11 January 2022; Accepted 15 January 2022; Published 7 February 2022

Academic Editor: Zhenzhou Yuan

Copyright (c) 2022 Mengyan Hu et al. This is an open access article distributed under the Creative Commons Attribution License, which permits unrestricted use, distribution, and reproduction in any medium, provided the original work is properly cited.

\begin{abstract}
The current platoon control strategies of connected autonomous vehicles (CAVs) focus on controlling the fixed intervehicle distance, i.e., the string stability of the platoon system. Here, we aimed to design a CAV platoon control strategy based on a constraint-following approach to solve the problem of platoon starting. As the resistance of the vehicle during driving varies with time, this study regarded the CAV platoon system as a changing dynamic system and introduced the Udwadia-Kalaba (U-K) approach to simplify the solution. Apart from adding an equality constraint, unlike most other studies, this study imposed a bilateral inequality constraint on the intervehicle distance between successive CAVs to prevent collisions. Meanwhile, a diffeomorphism method was introduced to transform the bounded state into an unbounded state. The proposed control strategy could render each CAV compliant with both the original imposed bilateral inequality constraint and the equality constraint. The former avoids collisions, and the latter indicates the string stability of the designed CAV platoon system. The effectiveness of the proposed controller was verified by numerical experiments. The gap errors tend to converge to zero, which is not amplified by the propagation of traffic flow.
\end{abstract}

\section{Introduction}

With the increasing growth of the automobile industry, urban transportation networks are experiencing significant issues in a variety of areas. It can easily cause speed breakdown, traffic flow oscillation, and congestion in converging portions of traffic flow in fragile highway systems for many cars [1]. In recent years, public interest in research on intelligent transportation systems has grown faster.

Autonomous vehicles are designed to free drivers from driving tasks and are expected to improve traffic safety and efficiency when connected through vehicle-to-vehicle (V2V) communication, i.e., connected autonomous vehicles (CAVs). They also have great potential for dealing with traffic problems $[2,3]$. Autonomous vehicles that use advanced sensing, communication, and control technologies have the potential to increase road capacity and improve traffic operations [4-6]. An adaptive cruise control system, one of the earliest autonomous vehicle systems, has already entered the market [7-9]. It uses on-board sensors to detect the surroundings and govern the speed of the vehicle to improve ride comfort. V2V and vehicle-to-infrastructure (V2I) communications enable CAVs to extend their visibility [10]. When a group of CAVs travels with a short intervehicle spacing or gap, a platoon is formed [11]. CAVs can share information and adopt more aggressive control strategies, thereby yielding better traffic throughput and increasing traffic efficiency $[12,13]$. In addition, driving in a platoon can change the aerodynamic forces on vehicles, thereby improving fuel economy [14-16]. Compared to individual autonomous vehicles, CAVs in a platoon have greater potential to improve traffic performance because they can share information and coordinate their behavior to ensure shorter intervehicle distances safely [4, 17-19], as illustrated in the field tests $[20,21]$. With V2I communication between roadside units and autonomous vehicles, traffic stability can be improved [22]. Hence, the study of the $\mathrm{CAV}$ platoon system is of great significance.

The earliest concept of an autonomous vehicle platoon system was proposed in 1939 [23], however, it has aroused 
great interest since 1986 [24]. Thereafter, an increasing number of studies on intelligent transportation systems have been conducted. Among them, control design is one of the most important issues in intelligent transportation systems. Stankovic et al. [25] proposed a decentralized overlapping control law for the platoons of intelligent vehicle systems. In particular, they adopted a reduced-order observer and feedback map to design the control law. Lee et al. [26] used a fuzzy sliding algorithm to build a platoon controller to compensate for the effect of uncertainty. Thus, the exact models of vehicles are not required. Antonelli et al. [27] separated the control architecture into two task-oriented stages. Therefore, the vehicle kinematic model is required to construct the control law to complete the task. Ghasemi et al. [28] proposed a hybrid controller consisting of a feedback linearization controller and a decentralized bidirectional controller for successive vehicles maintaining a constant space. Other recent studies can be found in [29-32]. Lee and Kim [33] present a control algorithm for a platoon of vehicles. The headway distance to the preceding vehicle and its changing rate along with the velocity of the leading vehicle are used to derive the headway control laws without using headway information from other vehicles. Desjardins and Chaib-Draa [34] proposed a novel approach for the design of autonomous vehicle controllers based on modern machinelearning techniques, which used function approximation techniques along with gradient-descent learning algorithms as a means of directly modifying a control policy to optimize its performance. To guarantee the string stability of a vehicle platoon, Kayacan [35] proposed a multiobjective $\mathrm{H}$-infinity control formulation for adaptive cruise control and cooperative adaptive cruise control structures. Wen et al. [36] proposed a useful string stable CCVP algorithm to guarantee the string stability and zero steady-state spacing error for the platoon, where the controller gain of the platoon is further complemented by additional conditions.

Platoon control strategy as one of the most significant aspects is studied by many researchers for decades. The motion of the CAV platoon in this study was regarded as a dynamic system because the aerodynamic resistance of each vehicle varied continuously, and it was necessary to dynamically adjust the tractive force of each vehicle to compensate for the changing resistance to ensure the stability of the platoon system. To control the dynamic system, Udwadia and Kalaba [37] developed a constraint-following approach, in which constraints are integrated into system dynamics in the form of "constraint force" and introduced a series of explicit and legible equations to solve the control law of a dynamic system subject to equality constraints. Because of its simplicity, the Udwadia-Kalaba (U-K) approach has been applied to various dynamic control problems and has shown good practicability [38-40]. However, the original $\mathrm{U}-\mathrm{K}$ approach was designed to cope with equality constraints. Hence, the problem of dealing with bilateral inequality constraints remains challenging [41].

To summarize, this study investigated the platoon control strategy for CAVs in the context of longitudinal motion. Our purpose was to design a CAV platoon control strategy, apply it to the platoon starting, and ensure the string stability of the CAV platoon under the premise of sufficient safety. To this end, we, firstly, formulated a nonlinear longitudinal dynamic model for each vehicle, considering the possible time-varying uncertainties, and we obtained a multivehicle dynamic system. Subsequently, we incorporated an equality constraint and a bilateral inequality constraint into the dynamic system. The former was set to ensure that spacing error between the current platoon state and the final platoon state would converge to zero. The latter was adopted to restrict the intervehicle distance within an appropriate range during the entire process to guarantee safety. The U-K approach was utilized to render an explicit control force for the specific dynamic system constructed in this study. As the original $U-K$ approach cannot handle inequality constraints, the diffeomorphism method [42] was applied to free the inequality constraint by transforming the bounded state to an unbounded state. Thus, the U-K approach could be used. Finally, the Lyapunov stability theory was employed to verify the performance of the proposed control strategy. We proved that the proposed control force could render uniform boundedness (UB) and uniform ultimate boundedness (UUB) performance for the unbounded state. The main difference between this study and other works is that the complicated platoon control problem was treated as a constraint-following problem, and the U-K approach was used to simplify the solution of this specific problem.

The remainder of this paper is organized as follows: the basic theory of the U-K approach is introduced in Section 2. The platoon model of a specific situation considering parameter uncertainty is detailed in Section 3. Subsequently, longitudinal dynamic models for a CAV platoon are introduced, and the control strategies based on the U-K approach are designed with and without parameter uncertainty in Section 4. The simulation experiments are designed to verify the performance of the controllers, followed by a discussion of the simulation results in Section 5 . Finally, the findings are concluded along with some future research directions in Section 6.

\section{U-K Approach}

In this section, we discuss the fundamental equations of the $\mathrm{U}-\mathrm{K}$ theory, which are the foundation of our research. This theory can be divided into the following three steps [37]:

2.1. Unconstrained System. In nature, all motions follow Newton's second law, and the generalized form of the equation of motion can be described as follows:

$$
F=m a,
$$

where $F$ is the total force exerted on the system, $m$ is the mass, and $a$ is the acceleration.

The Newton equation connects the force, mass, and acceleration of the system. Lagrange introduced the concept of generalized coordinates and used the D'Alembert principle to obtain an equation that is equivalent to Newton's second law. It is called the Lagrangian equation. The motion 
equation of $\mathrm{U}-\mathrm{K}$ mechanics for an unconstrained system can be obtained by Newtonian or Lagrangian mechanics, which can be written in the form of

$$
M(q, t) \ddot{q}=Q(q, \dot{q}, t),
$$

where $M$ is a positive definite inertia $n \times n$ matrix, $q$ is the coordinate and is an $\mathrm{n}$-vector, $\dot{q}$ is the velocity, $\ddot{q}$ is the acceleration, $t$ is the independent variable and generally refers to time, and $Q$ is the force [43] exerted on the system.

From (2), the generalized acceleration $a(q, \dot{q}, t)$ of the unconstrained system at time $t$ can be obtained.

$$
\ddot{q}=M^{-1}(q, t) Q(q, \dot{q}, t)=a(q, \dot{q}, t) .
$$

2.2. Constraints. There are inevitably some constraints presented in the system that need to be considered. These constraints can be roughly divided into two types: $\mathrm{m}$ holonomic and $n$ nonholonomic constraints. The former can perform second-order differentiation in the form of

$$
\varphi_{i}(q, t)=0, \quad(i=1,2 \ldots m),
$$

and the latter can perform first-order differentiation in the form of

$$
\varphi_{j}(q, \dot{q}, t)=0, \quad(j=1,2 \ldots n) .
$$

Given that (4) and (5) are sufficiently smooth and consistent, we can obtain the nominal generalized form of the constraints by differentiating $\varphi_{i}(q, t)$ twice and differentiating $\varphi_{j}(q, \dot{q}, t)$ once with regard to time $t$, which is given by

$$
A(q, \dot{q}, t) \ddot{q}=b(q, \dot{q}, t),
$$

where $A$ is a constraint matrix in the shape of $m \times n, \ddot{q}$ denotes the quadratic differential of the generalized coordinates, and $b$ is an $m$-dimensional vector.

Remark 1. It is undoubted that differential operations will lead to the loss of some information, such as constants. In fact, the initial condition of the system state usually satisfies zero-order or holonomic constraints, as shown in (4), which means that the lost information is retained in the initial condition. It was demonstrated in [40].

Remark 2. The constraint equation used in Lagrangian mechanics is in the form of zero-order (4) or first-order (5), whereas the $\mathrm{U}-\mathrm{K}$ equation is established based on the second-order constraints in the form of (6). It is decoupled and unaffected by Lagrangian multipliers, which simplifies the solution of the Lagrangian motion equation.

2.3. Constrained System. The equation of motion with constraints can be obtained by combining (2) and (6). Additional "generalized forces of constraints" are applied to the system. Therefore, the actual motion equation of the constrained system can be written as follows:

$$
M(q, t) \ddot{q}=Q(q, \dot{q}, t)+Q^{c}(q, \dot{q}, t),
$$

where $Q^{c}$ is the constraint force causing the change in acceleration, which can be regarded as a set of control forces acting on the unconstrained system.

In the Lagrangian motion equation, $Q^{c}$ represents the ideal constraints according to the D'Alembert principle. Nonideal constraints are usually not taken into consideration. In the $\mathrm{U}-\mathrm{K}$ equation, the ideal and nonideal constraints are considered in the system, and $Q^{c}$ can be written in the form of

$$
Q^{c}(q, \dot{q}, t)=Q_{i d}^{c}(q, \dot{q}, t)+Q_{\text {nid }}^{c}(q, \dot{q}, t),
$$

where $Q_{i d}^{c}$ and $Q_{\text {nid }}^{c}$ indicate the ideal and nonideal constraint force vectors, respectively.

Remark 3. In the U-K equation, if ideal constraints were the only constraints involved in the system, which means that the third term in (8) equals to zero $\left(Q_{\text {nid }}^{c}=0\right)$, (8) would be homogeneous with the Lagrangian motion equation.

Udwadia and Kalaba developed the nominal explicit expressions [44] of $Q_{i d}^{c}$ and $Q_{\text {nid }}^{c}$.

$$
\begin{aligned}
Q_{i d}^{c} & =M^{1 / 2} B^{+}(b-A a), \\
Q_{\text {nid }}^{c} & =M^{1 / 2}\left[I-(B)^{+} B\right] M^{-1 / 2} C,
\end{aligned}
$$

where $B=A M^{-1 / 2}, a=Q M^{-1}$, “+” represents "Moore-Penrose" generalized inverse [45], and $C$ is an $\mathrm{n}$-vector governed by the proposed system.

Upon substituting (8)-(11) in (7), the general equation of motion in $\mathrm{U}-\mathrm{K}$ mechanics can be obtained as follows:

$$
M \ddot{q}=Q+M^{1 / 2} B^{+}(b-A a)+M^{1 / 2}\left[I-(B)^{+} B\right] M^{-1 / 2} C .
$$

Remark 4. Udwadia and Kalaba demonstrated that the constraint force allows all the constraints to be strictly met at every moment with minimum control cost [37]. Lagrangian multipliers, which are always challenging to obtain, do not arise in the system motion (10). With the control input $\tau=Q^{c}$, system (10) satisfies constraint (6), including both ideal and nonideal constraints.

\section{Modelling of CAV Platoon System}

Consider that a platoon system consists of $\mathrm{n}$ vehicles, including a leading vehicle, and $n-1$ following vehicles in the same lane, as shown in Figure 1. This platoon system is homogeneous. It means that all the vehicles are of the same type. The red one is the leading vehicle, and the remaining $n-1$ white vehicles follow each other. These vehicles are all CAVs and are equipped with in-vehicle sensors, whose state information (such as velocity, acceleration, and location coordinates) can be measured during the driving process. In addition, these vehicles follow the predecessor-follower (PF) communication topology, which means that the preceding vehicle can send its state information exclusively to its closest follower. These $n$ vehicles are parking at these 


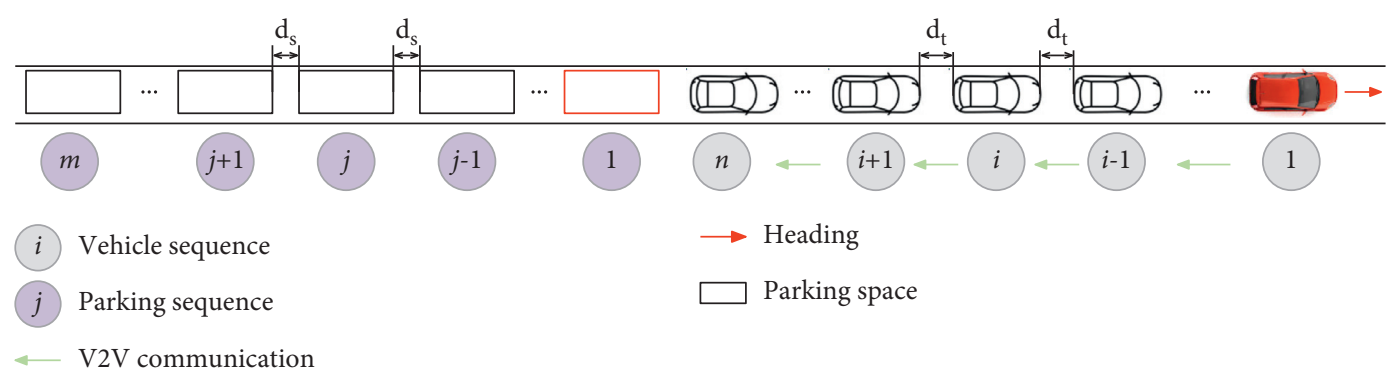

FIgURE 1: General platoon manoeuvre.

$m(m \geq n)$ designated parking spaces, and the red rectangle represents the first parking place, i.e., the parking place of the leading (red) vehicle. The remaining $n-1$ white vehicles are parking in $n-1$ black parking spaces behind the red vehicle. The parking distance between the $i^{\text {th }}$ and $i^{t h}+1$ vehicles is $d_{s}\left(d_{s} \leq d_{t}\right)$. During the normal operation of the platoon, it is considered that the distance between the $i^{\text {th }}$ and $i^{\text {th }}+1$ vehicles can regularly maintain $d_{t}$. When performing the starting process, the initial intervehicle distance $d_{s}$ will reach a string stability of intervehicle distance $d_{t}\left(d_{t} \neq d_{s}\right)$ as this process continues. In this section, the general platoon model is established based on nonlinear vehicle dynamics, constraints on the system, and possible time-varying parametric uncertainties.

3.1. Vehicle Dynamics Model. The position of the $k^{\text {th }}$ vehicle is denoted by $x_{k}$, based on geodetic coordinates. Therefore, the nonlinear longitudinal vehicle dynamic model for the $i^{\text {th }}(1 \leq i \leq n)$ vehicle can be described by

$$
\begin{aligned}
v_{i}(t) & =\dot{x}_{i}, \\
M_{i} \dot{v}_{i}(t) & =\tau_{i}-C_{i} v_{i}(t)\left|v_{i}(t)\right|-F_{i},
\end{aligned}
$$

where $t \in R$ denotes time, $M_{i} \in R$ represents the mass of the $i^{t h}$ vehicle, $v_{i} \in R$ is the velocity of the $i^{t h}$ vehicle at time $t$, $\tau_{i} \in R$ denotes the control input (i.e., the control law to be designed for the $i^{t h}$ vehicle), $-C_{i} v_{i}(t)\left|v_{i}(t)\right| \in R$ is the nominal aerodynamic resistance, and $F_{i} \in R$ is the nominal resistance force, acceleration resistance, and other external disturbances acting on the $i^{\text {th }}$ vehicle. The functions $C_{i}(\cdot)$ and $F_{i}(\cdot)$ are both continuous.

In a platoon system, the spacing between the two consecutive vehicles is the most noteworthy. The actual space between the $i^{\text {th }}$ vehicle and its preceding vehicle can be calculated as follows:

$$
d_{i}^{a s}(t)=x_{i-1}(t)-x_{i}(t)-l_{i-1},
$$

where $l_{i-1}$ denotes the length of the $i^{\text {th }}-1$ vehicle.

The desired space between the $i^{\text {th }}$ vehicle and its preceding vehicle is defined as $d_{i}^{d s}$. Thus, the space error can be calculated as follows:

$$
e_{i}(t)=d_{i}^{d s}-d_{i}^{a s}(t)=d_{i}^{d s}-x_{i-1}(t)+x_{i}(t)+l_{i-1} .
$$

For each vehicle in the platoon system, the adjustment of its own state depends not only on its own state but also on its position, speed, and acceleration. All of the above information can be obtained from (13). For example, $e_{i}$ contains position-related information, $\dot{e}_{i}$ speed-related information, and $\ddot{e}_{i}$ acceleration-related information because the position of the vehicle is regarded as continuous during driving. Therefore, $e_{i}$ is continuous and can perform a differential operation with respect to time $t$.

By substituting (13) in (11), the error dynamic equation for the $i^{\text {th }}$ vehicle is obtained, which is as follows:

$$
M_{i} \ddot{e}_{i}(t)=\tau_{i}-C_{i} v_{i}(t)\left|v_{i}(t)\right|-F_{i}-M_{i} x_{i-1}(t) .
$$

By rewriting (14) in the form of generalized U-K mechanics, we get

$$
M_{i} \ddot{e}_{i}(t)=Q_{i}(e, e, t)+Q_{i}^{c}(e, e, t)
$$

where $Q_{i}(e, e, t)=-C_{i} v_{i}(t)\left|v_{i}(t)\right|-F_{i}-M_{i} x_{i-1}(t)$ is the force exerted on the $i^{\text {th }}$ vehicle, and $Q_{i}^{c}(e, e, t)$ represents the control input $\tau_{i}$ mentioned in Remark 4, which is expected to be solved.

Remark 5. (15) is explicitly nonlinear because the resistance caused by air and road friction is nonlinear. In the present study, linearity can be treated as a special case of nonlinearity. Hence, all theories developed can be applied to linear systems as well.

3.2. Generic Constraints on the System. For this specific platoon-starting problem, we separate the overall constraints into two parts: constraints in the starting process and constraints after the starting process. For the former part, two situations should be avoided. (I) The $i^{\text {th }}$ vehicle starts slowly, and the $i^{t h}-1$ vehicle starts quickly, which may cause $d_{i}^{a s}$ to be too small or even zero (i.e., collision). (II) The $i^{\text {th }}$ vehicle starts quickly, and the $i^{\text {th }}-1$ vehicle starts slowly, which may cause $d_{i+1}^{a s}$ to be too small as a result of $d_{i}^{a s}$ being too large. To this end, $d_{i}^{a s}$ should be maintained within a reasonable range. Therefore, we have

$$
d_{\min } \leq d_{i}^{a s} \leq d_{\max } \longrightarrow d_{i}^{d s}-d_{\max } \leq e_{i}(t) \leq d_{i}^{d s}-d_{\min }
$$

For the constraints after the starting process, each vehicle is expected to travel at a constant car-following distance, i.e.,

$$
d_{i}^{a s}=d_{i}^{d s} \longrightarrow e_{i}(t)=0
$$


Apart from (17), the acceleration of each vehicle should be zero. Therefore, the following equality constraint is imposed on the platoon system.

$$
\eta_{i}^{1} e_{i}(t)+\eta_{i}^{2} \dot{e}_{i}(t)=0
$$

By solving (18), $e_{i}(t)$ can be obtained as follows:

$$
e_{i}(t)=e_{i}\left(t_{0}\right) \exp \left(-\frac{\eta_{i}^{1}}{\eta_{i}^{2}} t\right)
$$

Remark 6. $\eta_{i}^{1}$ and $\eta_{i}^{2}$ are scalar constants. It is clear that, for any given initial state $e_{i}\left(t_{0}\right), e_{i}(t)$ will converge to zero as $t$ is sufficiently long if constraint (18) is strictly satisfied. Moreover, $\dot{e}_{i}(t)$ will also converge to zero by adjusting $\eta_{i}^{1}$ and $\eta_{i}^{2}$. It should be noted that the position-related information and velocity-related information are contained in $e_{i}(t)$ and $\dot{e}_{i}(t)$, respectively. With this specific constraint imposed on the $i^{\text {th }}$ vehicle of the platoon system, when $e_{i}(t)$ and $\dot{e}_{i}(t)$ converge to zero, $\eta_{i}^{1} e_{i}(t)+\eta_{i}^{2} \dot{e}_{i}(t)=0$ will be satisfied. When $\eta_{i}^{1} e_{i}(t)+\eta_{i}^{2} \dot{e}_{i}(t)=0$ is achieved, both $e_{i}(t)$ and $\dot{e}_{i}(t)$ converge to zero.

By rewriting (18) in the form of (6), we get the following:

$$
A(e, \dot{e}, t) \ddot{e}=b(e, \dot{e}, t),
$$

where $A=1$ and $b=-\eta_{i}^{1} / \eta_{i}^{2} \dot{e}_{i}(t)$ refer to the specific problem in this study.

3.3. Generic Parameter Uncertainty. In this section, the parametric uncertainty is considered in system (14), where $C_{i}$ and $F_{i}$ can be redescribed [46] as follows:

$$
\begin{aligned}
\widetilde{C}_{i}(e, \dot{e}, \sigma, t) & =C_{i}(e, \dot{e}, t)+\Delta C_{i}(e, \dot{e}, \sigma, t), \\
\widetilde{F}_{i}(e, \dot{e}, \sigma, t) & =F_{i}(e, \dot{e}, t)+\Delta F_{i}(e, \dot{e}, \sigma, t),
\end{aligned}
$$

where $\Delta C_{i}$ and $\Delta F_{i}$ are uncertain portions, and $\sigma \in R^{p}$ is an uncertain parameter, which might vary with time. In the real world, $\sigma$ is always bounded. Therefore, $\Sigma$ is used to represent the possible bounding of $\sigma$. It is also assumed that the functions $\widetilde{C}_{i}, \Delta C_{i}, \widetilde{F}_{i}$, and $\Delta F_{i}$ are continuous. It should be noted that $\Sigma$ is tight but unknown.

By substituting (21) in (15), the motion equation considering parametric uncertainty can be rewritten as follows:

$$
M_{i} \ddot{e}_{i}(t)=\widetilde{Q}_{i}(e, e, t)+\widetilde{Q}_{i}^{c}(e, e, t),
$$

where $\widetilde{Q}_{i}=-\widetilde{C}_{i} v_{i}(t)\left|v_{i}(t)\right|-\widetilde{F}_{i}-M_{i} x_{i-1}(t)$.

\section{Vehicle Longitudinal Motion Controller}

In Section 3.2, constraint (16) is a bilateral inequality constraint and cannot be rewritten in the form of (6). Therefore, the U-K approach cannot be directly applied to solve this problem because the control force established by $\mathrm{U}-\mathrm{K}$ may violate (16) even if it satisfies (18). In this section, a model that converts bilateral inequalities into equations is introduced so that the U-K approach can be employed.
Afterward, the control with and without parameter uncertainty was designed and proved to be available.

4.1. Constraints Transfer. Diffeomorphism [41, 42, 47] is adopted to address this bilateral inequality problem because it can remove the inequality constraint (16) from the twoside-bounded space, thereby leading to an unbounded space. The definition of diffeomorphism [42] is expressed as follows: given two state spaces $X$ and $Y$, there is an invertible map $T: X \longrightarrow Y$ between them, and its inverse is $T^{-1}: Y \longrightarrow X$, as long as $T$ satisfies the following rules:

(I) Both $T$ and $T^{-1}$ are $\mathrm{k}$ times continuously differentiable.

(II) The correspondence between $X$ and $Y$ is exclusive.

The purpose is to choose an appropriate function $T$ to transform the bilateral inequality constraint from its original coordinate space $e_{i}$ to a new and unbounded coordinate space, which means that $T$ satisfies the following properties:

$$
\left\{\begin{array}{l}
e_{i} \in\left(d_{i}^{d s}-d_{\max }, d_{i}^{d s}-d_{\min }\right), \\
T: z_{i} \in R
\end{array}\right.
$$

It is obvious that the independent variable $e_{i}$ is bilateral bounded, and the variable $z_{i}$ after transformation is unbounded. It is noticeable that the sigmoid functions satisfy the property of (23). Therefore, we choose the following function $T$ for $e_{i}$ :

$$
T: z_{i}=\frac{1}{1+e^{k_{i}^{1} \cdot e_{i}+k_{i}^{2}}}+k_{i}^{3},
$$

where $k_{i}^{1}, k_{i}^{2}$, and $k_{i}^{3}$ are coefficients to be determined, $k_{i}^{1}$ and $k_{i}^{2}$ can be calculated by substituting (23) in (24), and $k_{i}^{3}$ represents the flexibility of diffeomorphism, which varies according to the specific situation of the control problem.

After transformation, $e_{i}$ can be rewritten as follows:

$$
e_{i}=T^{-1}\left(z_{i}\right) \text {. }
$$

By differentiating (25) once and twice, respectively, we get the following:

$$
\begin{aligned}
& \dot{e}_{i}=\frac{\partial T^{-1}\left(z_{i}\right)}{\partial z_{i}} \cdot \dot{z}_{i}, \\
& e_{i}=\frac{\partial^{2} T^{-1}\left(z_{i}\right)}{\partial z_{i}^{2}} \cdot \dot{z}_{i}^{2}+\frac{\partial T^{-1}\left(z_{i}\right)}{\partial z_{i}} \cdot z_{i} .
\end{aligned}
$$

By substituting (25) and (26) in (18), the constraints can be described as follows:

$$
T^{-1}\left(z_{i}\right)+\frac{\partial T^{-1}\left(z_{i}\right)}{\partial z_{i}} \cdot \dot{z}_{i}=0 .
$$

By rewriting (27) in the form of (20), we get the following:

$$
\hat{A}(z, \dot{z}, t) z=\widehat{b}(z, \dot{z}, t)
$$


where $\hat{A}=A \cdot \partial T^{-1}\left(z_{i}\right) / \partial z_{i}$ and $\hat{b}=b-A \partial^{2} T^{-1}\left(z_{i}\right) / \partial z_{i}^{2} \cdot \dot{z}_{i}^{2}$.

On account of the nature of diffeomorphism, the constraints of (28) are consistent and equivalent to (16) and (20), including only equality constraints regarding $z_{i}$. Therefore, the U-K approach can be directly applied to solve this problem.

4.2. Control without Parameter Uncertainty. In this study, it was assumed that, in system (15), the tires of each vehicle in the fleet do not slide relative to the ground. Therefore, no nonideal constraint is involved in this platoon system. Only ideal constraints are considered, and the motion equation of this platoon system regarding $z_{i}$ without parameter uncertainty considered can be expressed as follows:

$$
\hat{M} z=\widehat{Q}(z, \dot{z}, t)+\widehat{Q}^{c}(z, \dot{z}, t),
$$

where $\quad \hat{M}=M \cdot \partial T^{-1}\left(z_{i}\right) / \partial z_{i} \quad$ and $\quad \widehat{Q}=Q-M \cdot \partial^{2}$ $T^{-1}\left(z_{i}\right) / \partial z_{i}^{2} \cdot \dot{z}_{i}^{2}$

The explicit expression of the control force $\tau_{i}$ can be written as follows:

$$
\begin{gathered}
\widehat{Q}_{i}^{c}=\widehat{\tau}_{i}=\widehat{Q}_{i, i d}^{c}=\hat{M}^{1 / 2} \widehat{B}^{+}(\widehat{b}-\hat{A} \widehat{a}), \\
\sqrt{a^{2}+b^{2}},
\end{gathered}
$$

where $\widehat{B}=\hat{A} \hat{M}^{-1 / 2}$ and $\widehat{a}=\widehat{Q} \hat{M}^{-1}$

Theorem 1. If system (29) is subject to the constraint force in (30), then the motion of the system will satisfy the equality constraint in (28), i.e., $\hat{A} z=\widehat{b}$.

Proof: it is obvious that $\widehat{B}$ is one-dimensional in this particular problem and is also consistent. Therefore, we introduce the subsequent characteristics of the Moore-Penrose generalized inverse of $\widehat{B}$.

$$
\widehat{B} \widehat{B}^{+}=I \text {, }
$$

where $I$ is a unit matrix, which is one-dimensional of this specific situation.

Therefore, by combining system (29) and the constraint force (28), we get the following:

$$
\begin{aligned}
& \hat{A} z=\hat{A} \hat{M}^{-1}\left(\widehat{Q}+\widehat{Q}^{c}\right) \\
& =\hat{A} \hat{M}^{-1}\left(\widehat{Q}+\hat{M}^{1 / 2} \widehat{B}^{+}(\widehat{b}-\hat{A} \widehat{a})\right)
\end{aligned}
$$

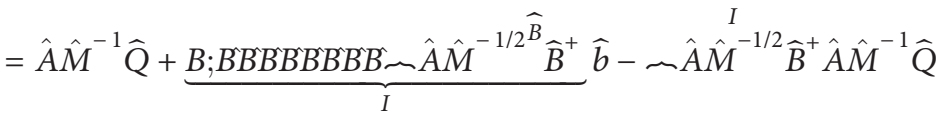

$$
\begin{aligned}
& =\hat{A} \hat{M}^{-1} \widehat{Q}+\widehat{b}-\hat{A} \hat{M}^{-1} \widehat{Q} \\
& =\widehat{b} \text {. }
\end{aligned}
$$

Because of the property of diffeomorphism, the equality constraint in state space $z_{i}$ has the same form as (18), i.e.,

$$
\eta_{i}^{1} z_{i}(t)+\eta_{i}^{2} \dot{z}_{i}(t)=0
$$

Our goal is to construct a feedback force $F_{2}$ that steers the actual position to meet the desired trajectory, i.e., the constraint (33). Here, we use $\beta_{i}$ to represent the deviation between the actual position and the desired position of the $i^{\text {th }}$ vehicle, which can be defined as follows:

$$
\lim _{t \longrightarrow t_{s}} \beta_{i}=\eta_{i}^{1} z_{i}(t)+\eta_{i}^{2} \dot{z}_{i}(t) \longrightarrow 0,
$$

where $t_{\mathrm{s}}$ is a large abstract value, which is determined according to the specific situation of the control problem.

The system will work as expected under the following control law.

$$
\widehat{Q}_{i}^{c}=F_{1}+F_{2} \text {, }
$$

where $F_{1}=\widehat{\tau}_{i}$ represents the constrained force, $F_{2}=\lambda_{i} \hat{M}_{i} \beta_{i}$ [42] indicates the feedback force, and $\lambda_{i}$ is a scalar constant.

By substituting (35) in (29), the motion equation of the $i^{\text {th }}$ vehicle can be obtained as follows:

$$
\begin{aligned}
z_{i} & =\hat{M}_{i}^{-1}\left(\widehat{Q}_{i}(z, \dot{z}, t)+\hat{M}_{i}^{1 / 2} \widehat{B}_{i}^{+}\left(\widehat{b}_{i}-\hat{A}_{i} \widehat{a}_{i}\right)+\lambda_{i} \hat{M}_{i} \beta_{i}\right) \\
& =\hat{A}_{i}^{-1} \widehat{b}_{i}\left(\frac{\eta_{i}^{1}}{\eta_{i}^{2}} \dot{z}_{i}\right)+\lambda_{i} \beta_{i} .
\end{aligned}
$$

We choose the following Lyapunov function candidate for the $i^{\text {th }}$ vehicle.

$$
V_{i}=\frac{1}{2} \hat{M}_{i} \beta_{i}^{2}
$$

By differentiating (37) and combining (34) and (36), we get the following:

$$
\begin{aligned}
\dot{V}_{i} & =\hat{M}_{i} \beta_{i} \dot{\beta}_{i} \\
& =\hat{M}_{i} \beta_{i}\left(\eta_{i}^{1} \dot{z}_{i}(t)+\eta_{i}^{2} z_{i}(t)\right) \\
& =\lambda_{i} \hat{M}_{i} \beta_{i}^{2} .
\end{aligned}
$$

It is explicit that $V_{i}$ is positive definite, and $\dot{V}_{i}$ is negative definite in view of $\lambda_{i}<0 . \dot{V}_{i}=0$ if and only if $\beta_{i}=0$. Therefore, systems (29) and (28) are asymptotically stable. 
4.3. Control with Parameter Uncertainty. By substituting (21) in (22), we obtain the overall external force exerted on the vehicle (by nature) that considers time-varying parameter uncertainty.

$$
\begin{aligned}
\widetilde{Q}_{i} & =-\widetilde{C}_{i} v_{i}(t)\left|v_{i}(t)\right|-\widetilde{F}_{i}-M_{i} x_{i-1}(t) \\
& =-\left(C_{i}(e, \dot{e}, t)+\Delta C_{i}(e, \dot{e}, \sigma, t)\right) v_{i}(t)\left|v_{i}(t)\right|-\left(F_{i}(e, \dot{e}, t)+\Delta F_{i}(e, \dot{e}, \sigma, t)\right)-M_{i} x_{i-1}(t) \\
& =-M_{i} x_{i-1}(t)-C_{i}(e, \dot{e}, t) v_{i}(t)\left|v_{i}(t)\right|-F_{i}(e, \dot{e}, t)-\Delta C_{i}(e, \dot{e}, \sigma, t) v_{i}(t)\left|v_{i}(t)\right|-\Delta F_{i}(e, \dot{e}, \sigma, t) .
\end{aligned}
$$

By substituting (26) and (39) in (22), the motion equation with parameter uncertainty can be obtained as follows:

$$
\begin{aligned}
z_{i}= & -\frac{\partial T^{-1}\left(z_{i}\right)^{-1}}{\partial z_{i}} x_{i-1}(t)-\frac{\partial T^{-1}\left(z_{i}\right)^{-1}}{\partial z_{i}} \frac{\partial^{2} T^{-1}\left(z_{i}\right)}{\partial z_{i}^{2}} \cdot \dot{z}_{i}^{2} \\
& +\left(M \cdot \frac{\partial T^{-1}\left(z_{i}\right)}{\partial z_{i}}\right)^{-1}\left[-C_{i} v_{i}(t)\left|v_{i}(t)\right|-F_{i}-\Delta C_{i} v_{i}(t)\left|v_{i}(t)\right|-\Delta F_{i}+\widetilde{Q}_{i}^{c}\right] \\
= & \left(M \cdot \frac{\partial T^{-1}\left(z_{i}\right)}{\partial z_{i}}\right)^{-1} \widetilde{Q}_{i}^{c}-\frac{\partial T^{-1}\left(z_{i}\right)^{-1}}{\partial z_{i}} x_{i-1}(t)-\frac{\partial T^{-1}\left(z_{i}\right)^{-1}}{\partial z_{i}} \frac{\partial^{2} T^{-1}\left(z_{i}\right)}{\partial z_{i}^{2}} \cdot \dot{z}_{i}^{2} \\
& -\left(M \cdot \frac{\partial T^{-1}\left(z_{i}\right)}{\partial z_{i}}\right)^{-1}\left[C_{i}\left(\frac{\partial T^{-1}\left(z_{i}\right)}{\partial z_{i}} \cdot \dot{z}_{i}+\dot{x}_{i-1}(t)\right)\left|\frac{\partial T^{-1}\left(z_{i}\right)}{\partial z_{i}} \cdot \dot{z}_{i}+\dot{x}_{i-1}(t)\right|+F_{i}\right] \\
& -\left(M \cdot \frac{\partial T^{-1}\left(z_{i}\right)}{\partial z_{i}}\right)^{-1}\left[\Delta C_{i}\left(\frac{\partial T^{-1}\left(z_{i}\right)}{\partial z_{i}} \cdot \dot{z}_{i}+\dot{x}_{i-1}(t)\right)\left|\frac{\partial T^{-1}\left(z_{i}\right)}{\partial z_{i}} \cdot \dot{z}_{i}+\dot{x}_{i-1}(t)\right|+\Delta F_{i}\right] .
\end{aligned}
$$

In this section, the parameter uncertainty is considered to build the control law. As mentioned in (35) of Section 4.2, the control law contains two parts: constraint force and feedback force. In this section, an additional force should be applied to eliminate the parameter uncertainty, i.e., the last term in (40). Therefore, the control law considering parameter uncertainty is defined as follows:

$$
\widetilde{Q}_{i}^{c}=F_{1}+F_{2}+F_{3}
$$

where $F_{3}$ is the so-called adaptive control force, which aims to compensate for parameter uncertainty, $F_{1}$ is constraint force, and $F_{1}=\widehat{\tau}_{i}$ still holds, i.e.,

$$
\begin{aligned}
& F_{1}=\hat{M}^{1 / 2} \widehat{B}^{+}(\hat{b}-\hat{A} \widehat{a}) \\
& =\hat{M}^{1 / 2} \widehat{B}^{+}\left(-\frac{\eta_{i}^{1}}{\eta_{i}^{2}} \dot{z}_{i}-\hat{A} \widehat{Q} \hat{M}^{-1}\right) \\
& =-\hat{M} \frac{\eta_{i}^{1}}{\eta_{i}^{2}} \dot{z}_{i}-\widehat{Q}+M \cdot \frac{\partial^{2} T^{-1}\left(z_{i}\right)}{\partial z_{i}^{2}} \cdot \dot{z}_{i}^{2} \\
& =-\hat{M} \frac{\eta_{i}^{1}}{\eta_{i}^{2}} \dot{z}_{i}+C_{i} v_{i}(t)\left|v_{i}(t)\right|+F_{i} \\
& +M_{i} x_{i-1}(t)+M \cdot \frac{\partial^{2} T^{-1}\left(z_{i}\right)}{\partial z_{i}^{2}} \cdot \dot{z}_{i}^{2} .
\end{aligned}
$$

Identically, $F_{2}$ has the same form as in (35).

$$
F_{2}=\lambda_{i} \hat{M}_{i} \beta_{i}
$$

In Section 4.2, we demonstrated that $F_{1}+F_{2}$ can stabilize the platoon control system. Therefore, the purpose of this section is to construct the adaptive control force $F_{3}$ to handle the time-varying parameter uncertainty.

In Section 3.3, we mentioned the relationship between $\sigma$ and $\Sigma$. Hence, the following assumption is introduced.

Assumption 1. There exists a known function $\prod_{i}(\cdot): R \times R \times R \times R \longrightarrow R_{+}$, when $\sigma_{i} \in \Sigma_{i}$, for all vectors $\left(z_{i}, \dot{z}_{i}, \sigma_{i}, t\right) \in R \times R \times R \times R$, such that

$$
\begin{gathered}
\max _{\sigma_{i} \in \Sigma_{i}} \mid\left(M \cdot \frac{\partial T^{-1}\left(z_{i}\right)}{\partial z_{i}}\right)^{-1}\left[\Delta C_{i}\left(\frac{\partial T^{-1}\left(z_{i}\right)}{\partial z_{i}} \cdot \dot{z}_{i}+\dot{x}_{i-1}(t)\right)\right. \\
\left.\left|\frac{\partial T^{-1}\left(z_{i}\right)}{\partial z_{i}} \cdot \dot{z}_{i}+\dot{x}_{i-1}(t)\right|+\Delta F_{i}\right] \mid \leq \Pi_{i}\left(z_{i}, \dot{z}_{i}, \sigma_{i}, t\right) .
\end{gathered}
$$

Remark 7. (44) represents the parameterization of the worst-case effect of uncertainty. As mentioned in Section 3.3, the specific parameter uncertainty is unknown, whereas its boundary can be restricted by the known function $\Pi_{i}(\cdot)$ 
We now propose the adaptive control force $F_{3}$ as follows [42]:

$$
F_{3}=\hat{M}_{i} \gamma_{i} \mu_{i} \Pi_{i}
$$

where,

$$
\begin{aligned}
\gamma_{i} & = \begin{cases}\frac{1}{\left|\mu_{i}\right|}, & \text { if }\left|\mu_{i}\right|>\epsilon_{1}, \\
\frac{1}{\epsilon_{i}}, & \text { if }\left|\mu_{i}\right| \leq \epsilon_{i},\end{cases} \\
\mu_{i}=\beta_{i} \Pi_{i} & \\
= & \left(\eta_{i}^{1} z_{i}(t)+\eta_{i}^{2} \dot{z}_{i}(t)\right) \Pi_{i},
\end{aligned}
$$

and $\epsilon_{i}>0$ is a scalar constant.
Theorem 2. Considering Assumption 1 and the system motion (40), the adaptive control (41) yields the following performance [41, 43]:

(I) UB: for any $r_{i}>0$, there exists $d_{i}\left(r_{i}\right)<\infty$ such that, if $\beta_{i}$ is any solution with $\beta_{i}\left(t_{0}\right) \leq r_{i}$, then $\beta_{i}(t) \leq d_{i}\left(r_{i}\right)$ for all $t \geq t_{0}$.

(II) UUB: for any $r_{i}>0$ with $\beta_{i}\left(t_{0}\right) \leq r_{i}$, there exists $d_{i}>0$ such that $\beta_{i}(t) \leq \overline{d_{i}}$ for any $\overline{d_{i}}>d_{i}$ as $\overline{t \geq} t_{0}+T\left(\bar{d}_{i}, r_{i}\right)$, where $T\left(\bar{d}_{i}, r_{i}\right)<\infty$.

Proof: . similar to (37), we choose the Lyapunov function candidate.

$$
V_{i}=\frac{1}{2} \hat{M}_{i} \beta_{i}^{2} .
$$

Differentiating (47) and combining (34) and (40), we obtain the derivative of $V_{i}$ in the form of (48).

$$
\begin{aligned}
& \dot{V}_{i}=\hat{M}_{i} \beta_{i}\left(\eta_{i}^{1} \dot{z}_{i}(t)+\eta_{i}^{2} z_{i}(t)\right) \\
& =\frac{\beta_{i}}{\eta_{i}^{2}}\left(\frac{\eta_{i}^{1}}{\eta_{i}^{2}} M_{i} \frac{\partial T^{-1}\left(z_{i}\right)}{\partial z_{i}} \dot{z}_{i}(t)+\widetilde{Q}_{i}^{c}-M_{i} x_{i-1}(t)-M_{i} \frac{\partial^{2} T^{-1}\left(z_{i}\right)}{\partial z_{i}^{2}} \cdot \dot{z}_{i}^{2}\right. \\
& -\left[C_{i}\left(\frac{\partial T^{-1}\left(z_{i}\right)}{\partial z_{i}} \cdot \dot{z}_{i}+\dot{x}_{i-1}(t)\right)\left|\frac{\partial T^{-1}\left(z_{i}\right)}{\partial z_{i}} \cdot \dot{z}_{i}+\dot{x}_{i-1}(t)\right|+F_{i}\right] \\
& \left.-\left[\Delta C_{i}\left(\frac{\partial T^{-1}\left(z_{i}\right)}{\partial z_{i}} \cdot \dot{z}_{i}+\dot{x}_{i-1}(t)\right)\left|\frac{\partial T^{-1}\left(z_{i}\right)}{\partial z_{i}} \cdot \dot{z}_{i}+\dot{x}_{i-1}(t)\right|+\Delta F_{i}\right]\right) \\
& =\frac{\beta_{i}}{\eta_{i}^{2}}\left[\frac{\eta_{i}^{1}}{\eta_{i}^{2}} M_{i} \frac{\partial T^{-1}\left(z_{i}\right)}{\partial z_{i}} \dot{z}_{i}(t)-M_{i} x_{i-1}(t)-M_{i} \frac{\partial^{2} T^{-1}\left(z_{i}\right)}{\partial z_{i}^{2}} \cdot \dot{z}_{i}^{2}\right. \\
& \left.-C_{i}\left(\frac{\partial T^{-1}\left(z_{i}\right)}{\partial z_{i}} \cdot \dot{z}_{i}+\dot{x}_{i-1}(t)\right)\left|\frac{\partial T^{-1}\left(z_{i}\right)}{\partial z_{i}} \cdot \dot{z}_{i}+\dot{x}_{i-1}(t)\right|-F_{i}+F_{1}\right] \\
& +\frac{\beta_{i}}{\eta_{i}^{2}}\left[-\Delta C_{i}\left(\frac{\partial T^{-1}\left(z_{i}\right)}{\partial z_{i}} \cdot \dot{z}_{i}+\dot{x}_{i-1}(t)\right)\left|\frac{\partial T^{-1}\left(z_{i}\right)}{\partial z_{i}} \cdot \dot{z}_{i}+\dot{x}_{i-1}(t)\right|-\Delta F_{i}\right] \\
& +\frac{\beta_{i}}{\eta_{i}^{2}} F_{2}+\frac{\beta_{i}}{\eta_{i}^{2}} F_{3}=J_{1}+J_{2}+J_{3}+J_{4} .
\end{aligned}
$$

It can be noticed that there are four terms in (48), and $J_{i}(i=1,2,3,4)$ correspond to them. By substituting (42) into $J_{1}$, we get the following:

$$
J_{1}=0 \text {. }
$$

By subjecting this to Assumption 1, we have

$$
J_{2} \leq\left|\frac{\beta_{i}}{\eta_{i}^{2}} \cdot M \cdot \frac{\partial T^{-1}\left(z_{i}\right)}{\partial z_{i}}\right| \Pi_{i}\left(z_{i}, \dot{z}_{i}, \sigma_{i}, t\right) .
$$

By substituting (43) into $J_{3}$, we have

$$
J_{3}=\frac{1}{\eta_{i}^{2}} \lambda_{i} \hat{M}_{i} \beta_{i}^{2}
$$


By combining (45) and (46), we get

$$
\begin{aligned}
J_{4} & =\frac{\beta_{i}}{\eta_{i}^{2}} \hat{M}_{i} \gamma_{i} \mu_{i} \Pi_{i} \\
& =\frac{\mu_{i}^{2}}{\eta_{i}^{2}} \hat{M}_{i} \gamma_{i}, \\
& = \begin{cases}\frac{\left|\mu_{i}\right|}{\eta_{i}^{2}} \hat{M}_{i}, & \text { if }\left|\mu_{i}\right|>\varepsilon_{i}, \\
\frac{\mu_{i}^{2}}{\varepsilon_{i} \eta_{i}^{2}} \hat{M}_{i}, & \text { if }\left|\mu_{i}\right| \leq \varepsilon .\end{cases}
\end{aligned}
$$

By combining (48)-(52) for $\left|\mu_{i}\right|>\varepsilon_{i}$, we get

$$
\begin{aligned}
\dot{V}_{i} \leq & \frac{1}{\eta_{i}^{2}} \lambda_{i} \hat{M}_{i} \beta_{i}^{2}-\left|\frac{\beta_{i}}{\eta_{i}^{2}} \cdot M \cdot \frac{\partial T^{-1}\left(z_{i}\right)}{\partial z_{i}}\right| \Pi_{i}\left(z_{i}, \dot{z}_{i}, \sigma_{i}, t\right) \\
& +\frac{\left|\mu_{i}\right|}{\eta_{i}^{2}} \hat{M}_{i}=\frac{1}{\eta_{i}^{2}} \lambda_{i} \hat{M}_{i} \beta_{i}^{2} ;
\end{aligned}
$$

for $\left|\mu_{i}\right|<\epsilon_{i}$ we get

$$
\begin{aligned}
\dot{V}_{i} \leq & \frac{1}{\eta_{i}^{2}} \lambda_{i} \hat{M}_{i} \beta_{i}^{2}-\left|\frac{\beta_{i}}{\eta_{i}^{2}} \cdot M \cdot \frac{\partial T^{-1}\left(z_{i}\right)}{\partial z_{i}}\right| \Pi_{i}\left(z_{i}, \dot{z}_{i}, \sigma_{i}, t\right) \\
& +\frac{\mu_{i}^{2}}{\epsilon_{i} \eta_{i}^{2}} \hat{M}_{i}=\frac{1}{\eta_{i}^{2}} \lambda_{i} \hat{M}_{i} \beta_{i}^{2}-\frac{\epsilon_{i}}{4 \eta_{i}^{2}} \hat{M}_{i} .
\end{aligned}
$$

Since $\varepsilon_{i}>0$, the following equation is satisfied for all $\left|\mu_{i}\right|$.

$$
\dot{V}_{i} \leq \frac{1}{\eta_{i}^{2}} \lambda_{i} \hat{M}_{i} \beta_{i}^{2}-\frac{\epsilon_{i}}{4 \eta_{i}^{2}} \hat{M}_{i}
$$

By referring to the standard arguments of [43], the UB of the system can be obtained as follows:

$$
d_{i}\left(r_{i}\right)= \begin{cases}R_{i}, & \text { if } r_{i} \leq R_{i}, \\ r_{i}, & \text { if } r_{i}>R_{i},\end{cases}
$$

where $R_{i}=\sqrt{\varepsilon_{i} / 4 \eta_{i}^{2} \hat{M}_{i}}$.

Furthermore, UUB mentioned in [42] also follows with the following:

$$
\begin{aligned}
\overline{d_{i}} & =R_{i}, \\
T\left(\bar{d}_{i}, r_{i}\right) & = \begin{cases}0, & \text { if } r_{i}<\bar{d}_{i} \\
r_{i}^{2}-\bar{d}_{i}^{2}, & \text { otherwise } \\
2 \lambda_{i} \bar{d}_{i}^{2}-\frac{\epsilon_{i}}{2 \eta_{i}^{2}} \hat{M}_{i}, & \end{cases}
\end{aligned}
$$

Remark 8. It is clear that $\lambda_{\mathrm{i}}$ and $\epsilon_{\mathrm{i}}$ are scalar constants, and $\dot{V}_{i}$ is negative for a sufficiently large $\left|\beta_{i}\right|$. It means that the deviation $\beta_{\mathrm{i}}$ will reduce once it becomes sufficiently large. The boundary of $\beta_{i}$ is determined by $\eta_{i}^{2}$ and $\hat{M}_{i}$, and the control parameters may determine the sizes of UB and UUB.

\section{Numerical Simulation}

In this section, simulations are performed in MATLAB to verify the effectiveness of the proposed platoon control algorithm. The simulations are divided into two categories corresponding to sections 5.1 and 5.2, respectively, i.e., the simulation without parameter uncertainty and the simulation with parameter uncertainty. It is also assumed that all the vehicles are CAVs and deployed with the same controller as proposed in this study, and the leading vehicle is driven by humans. For each vehicle in the CAV platoon, their workflow is shown in Figure 2.

5.1. Simulation Results without Parameter Uncertainty. In this section, we enforce the proposed controller (35) on the proposed platoon system without parameter uncertainty. The tire friction coefficient and resistance force are regarded as scalar constants and are listed in Table 1 along with some relevant parameters.

In this case study, the initial speed of the CAV platoon was $0 \mathrm{~km} / \mathrm{h}$, and it gradually increased to $100 \mathrm{~km} / \mathrm{h}$. The desired intervehicle distance $d_{t}$ was set to $15 \mathrm{~m}$. The inequality constraint for the intervehicle distance was $[12 \mathrm{~m}$, $18 \mathrm{~m}$ ]. The errors of the initial intervehicle distance between consecutive CAVs were randomly chosen in the range $[-2 \mathrm{~m}, 2 \mathrm{~m}]$. As mentioned above, the leading vehicle is driven by humans and has a constant acceleration of $2 \mathrm{~m} / \mathrm{s}^{2}$. The performances of other CAVs in the platoon under the proposed controller (38) are shown in Figures 3-6.

As shown in Figure 3, the initial intervehicle distance between consecutive vehicles is chaotic, with larger and smaller ones. As this process continues, the distance between them gradually becomes stable, which means that the CAV platoon under the proposed controller (35) will reach string stability.

Similar to the trajectories in Figure 3, Figure 4 shows the historical difference between the actual intervehicle distance and the desired intervehicle distance of each CAV. It can be noticed that each spacing error finally converges to zero, meaning that the gap error is eliminated by the proposed controller (35). Under the premise of ensuring safety, the spacing errors of all vehicles were mainly eliminated after approximately $9 \mathrm{~s}$. Furthermore, in the process of eliminating the spacing error, the driving state of each CAV tends to be homogeneous. As shown in Figure 5, the speed of each CAV also becomes almost the same after approximately $9 \mathrm{~s}$, which proves the efficiency of the proposed controller (35).

5.2. Simulation Results with Parameter Uncertainty. As mentioned above, the parameter uncertainty is unknown, however, its bounds meet Assumption 1. In the next simulations, it is assumed that Assumption 1 is satisfied with the following:

$$
\Pi_{i}\left(z_{i}, \dot{z}_{i}, \sigma_{i}, t\right)=0.5 \cdot \dot{z}_{i}+0.05 \cdot z_{i} .
$$

In addition, the possible time-varying parameter uncertainty, as mentioned in Section 3.3, always occurs in the tire 


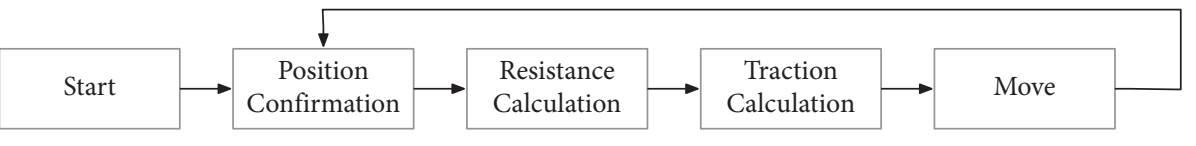

FIgUre 2: Workflow of the vehicle in the CAV platoon.

TABle 1: Parameters of the vehicles in platoon.

\begin{tabular}{lccc}
\hline$M(\mathrm{~kg})$ & $C\left(\mathrm{~N} \cdot \mathrm{s}^{2} / \mathrm{m}^{2}\right)$ & $F(\mathrm{~N})$ & $l(\mathrm{~m})$ \\
\hline 1500 & 0.5 & 300 & 4.6 \\
\hline
\end{tabular}

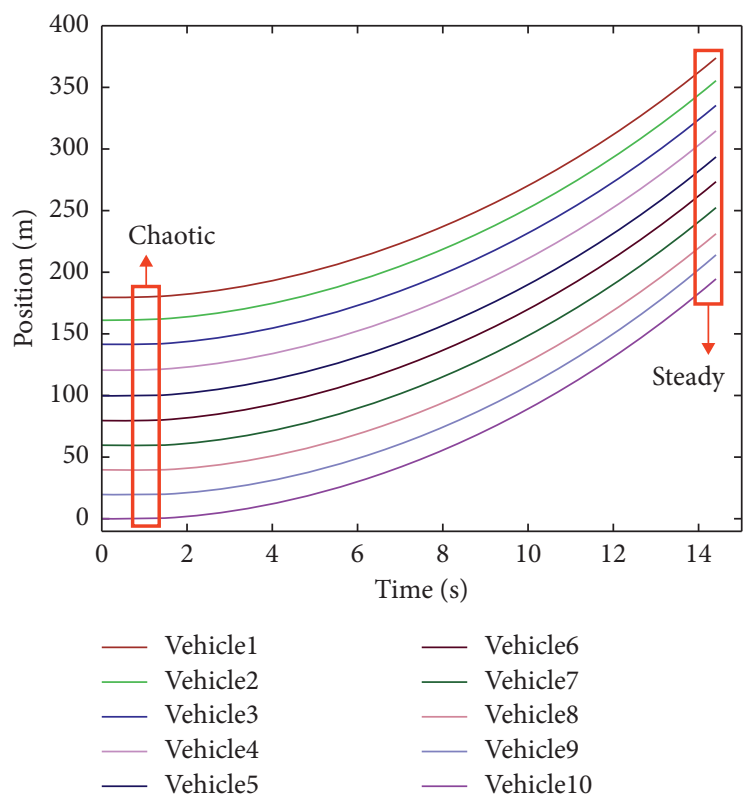

FIgURe 3: Position history under proposed controller (35).

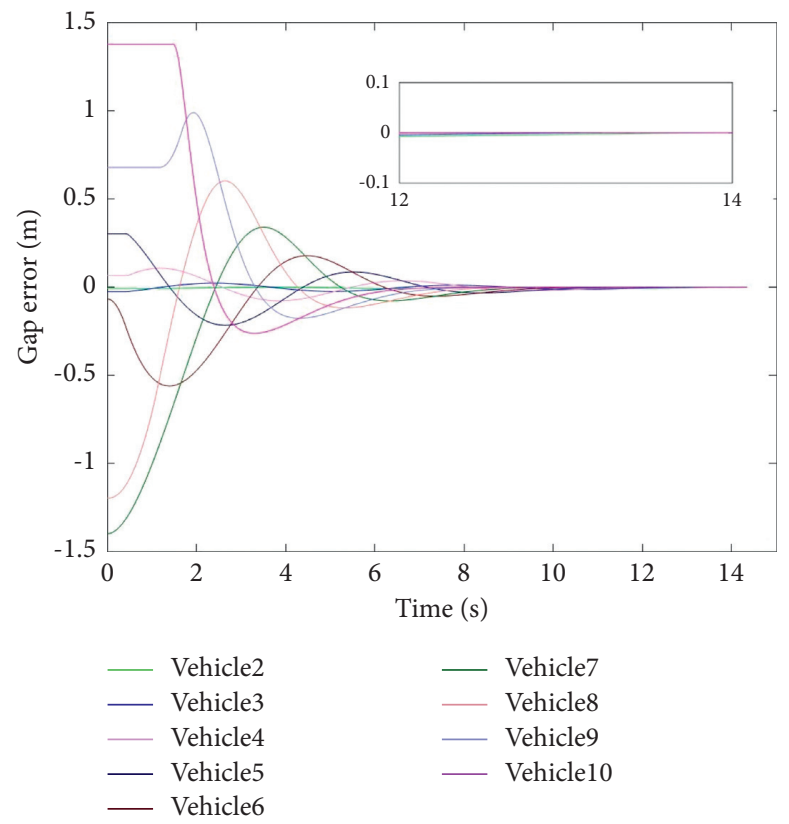

FIgURE 4: Gap error history under proposed controller (35). 


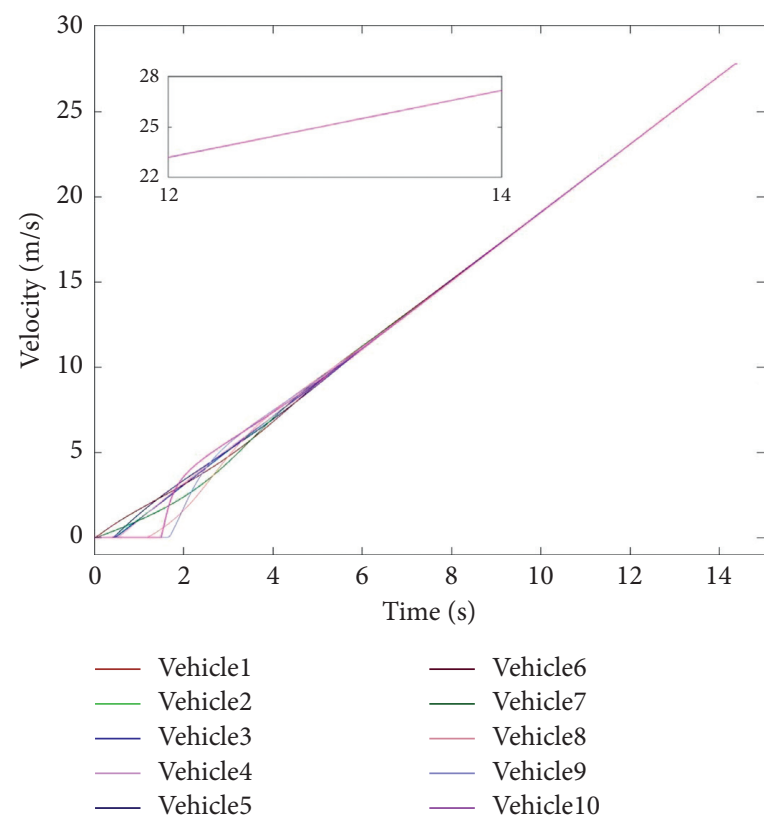

FIgURe 5: Velocity history under proposed controller (35).

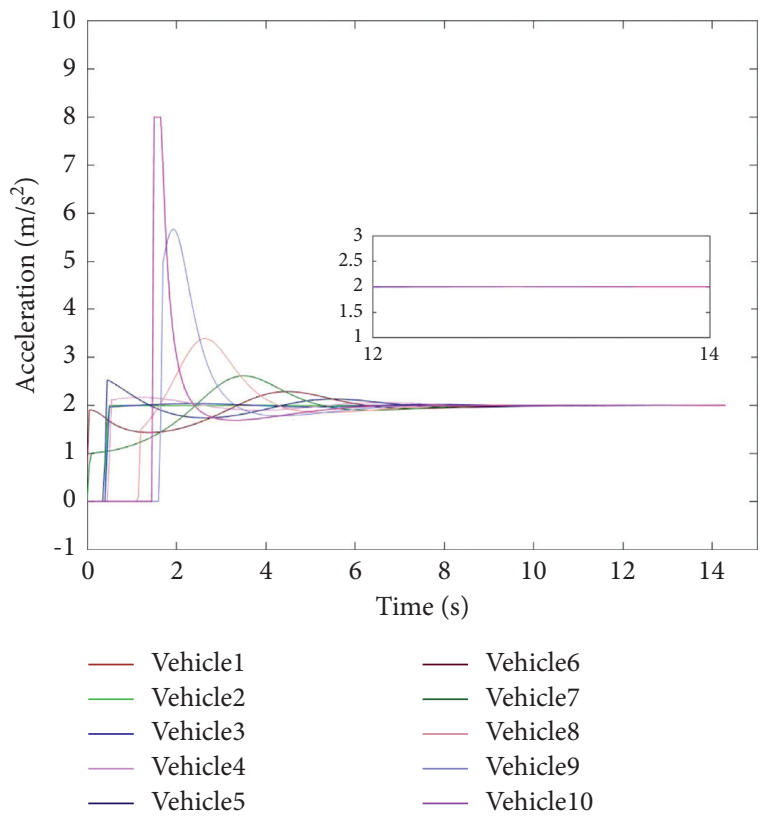

FIgURE 6: Acceleration history under proposed controller (35).

friction coefficient and resistance force. We randomly choose its values, which are listed in Table 2. Uncertainty for each vehicle in the platoon is implemented with a random initial phase in the following simulations. The friction coefficient of the ground or the air received by the vehicle varies in real-time during the vehicle's driving process, implying that the thinness of the air around the car is changing, as are the ground conditions. These changes are generally limited to a range. Hence, we simulate this range of change with a bounded function. The other settings are the same as those in Section 5.1.
These experimental results are similar to those of case 1 in Section 5.1. It can be observed from Figure 7 that the intervehicle distance between consecutive CAVs is gradually adjusted to approach the desired traveling intervehicle distance $d_{t}$. As shown in Figure 8, after $9 \mathrm{~s}$, each spacing error is mostly eliminated. Furthermore, each CAV in the platoon strictly follows the bilateral inequality constraint in this process, which fully guarantees driving safety. Moreover, the driving state of each CAV also tends to be the same, as shown in Figure 9. 
TABle 2: Parameters' uncertainty.

\begin{tabular}{lcr}
\hline Index & $\Delta C_{i}(\mathrm{~N} \cdot \mathrm{s} 2 / \mathrm{m} 2)$ & $\Delta F_{i}(\mathrm{~N})$ \\
\hline $\mathbf{1}$ & $0.5 \sin (0.1 t)$ & $300 \sin (t+3 \pi / 4)$ \\
$\mathbf{2}$ & $0.5 \cos (0.2 t+\pi / 4)$ & $300 \sin (0.9 t-5 \pi / 4)$ \\
$\mathbf{3}$ & $0.5 \sin (0.3 t-1 \pi / 2)$ & $300 \sin (0.8 t+3 \pi / 2)$ \\
$\mathbf{4}$ & $0.5 \sin (0.4 t+3 \pi / 4)$ & $300 \cos (0.7 t-7 \pi / 4)$ \\
$\mathbf{5}$ & $0.5 \sin (0.5 t-\pi)$ & $300 \sin (0.6 t)$ \\
$\mathbf{6}$ & $0.5 \cos (0.6 t+5 \pi / 4)$ & $300 \sin (0.5 t-\pi / 4)$ \\
$\mathbf{7}$ & $0.5 \cos (0.7 t+3 \pi / 2)$ & $300 \sin (0.4 t-\pi / 2)$ \\
$\mathbf{8}$ & $0.5 \cos (0.8 t+7 \pi / 4)$ & $300 \cos (0.3 t+3 \pi / 4)$ \\
$\mathbf{9}$ & $0.5 \sin (0.9 t)$ & $300 \sin (0.2 t-\pi)$ \\
$\mathbf{1 0}$ & $0.5 \sin (t-\pi / 4)$ & $300 \cos (0.1 t+5 \pi / 4)$ \\
\hline
\end{tabular}

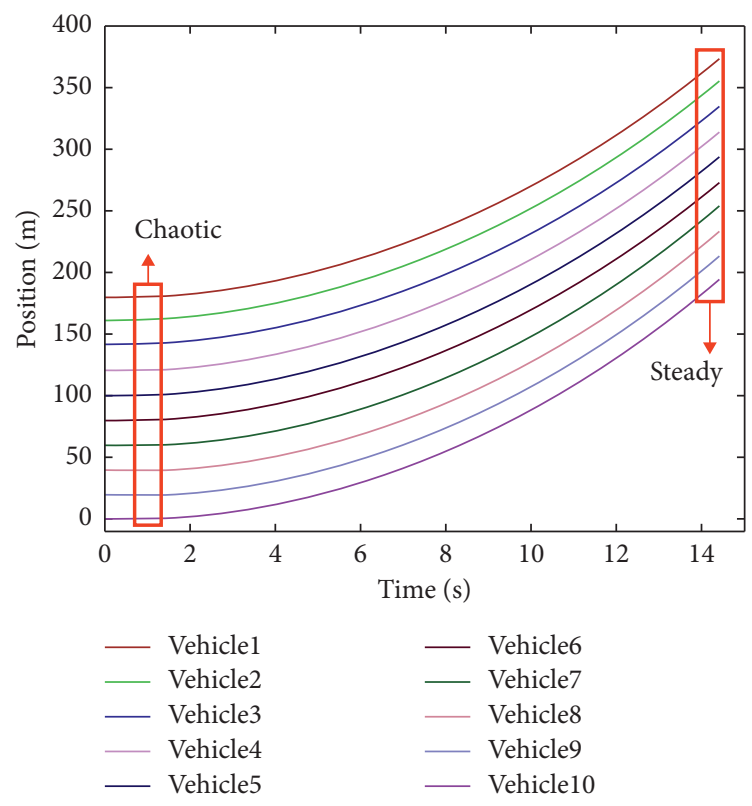

FIgURe 7: Position history under proposed controller (41).

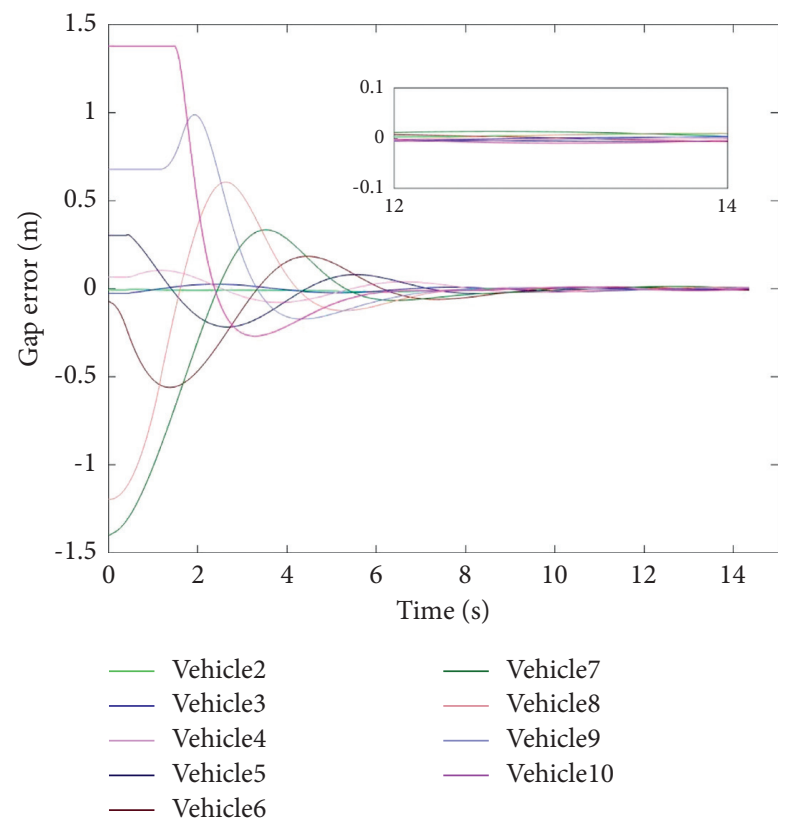

FIgURE 8: Gap error history under proposed controller (41). 


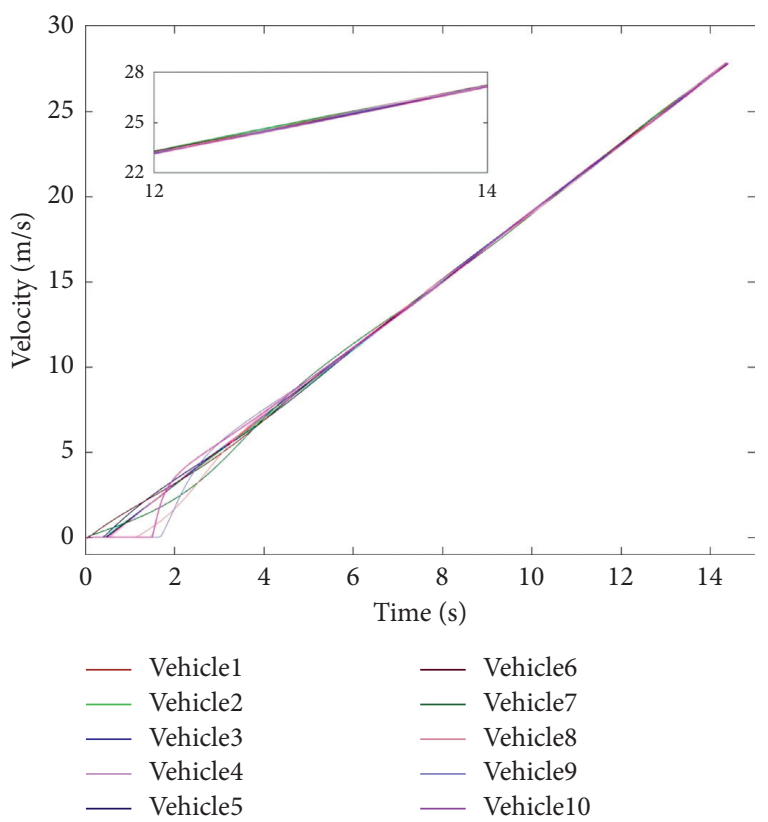

FIGURE 9: Velocity history under proposed controller (41).

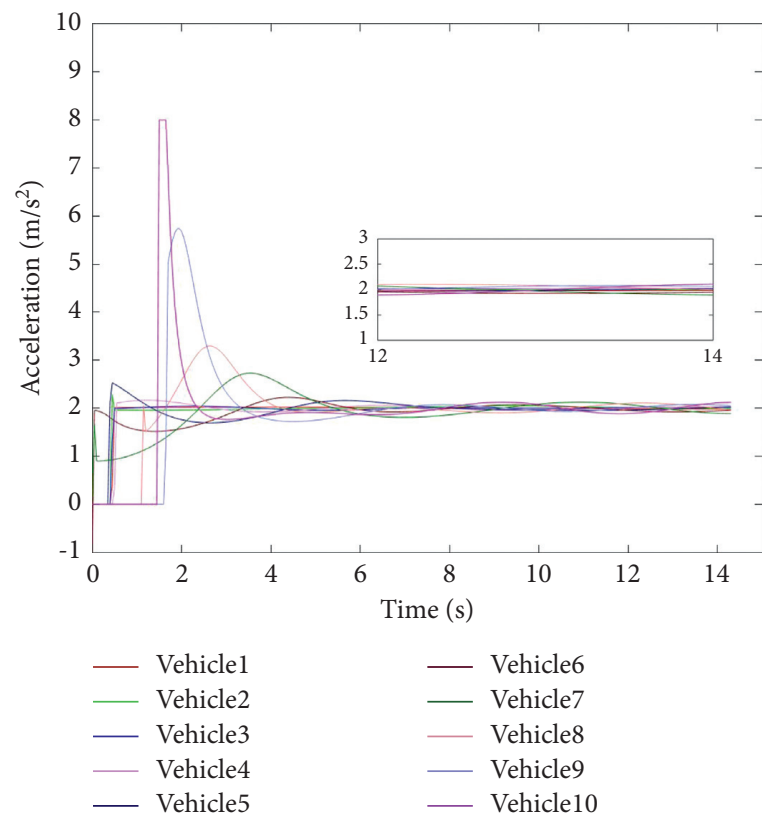

Figure 10: Acceleration history under proposed controller (41).

In contrast to case 2 in section 5.2, fluctuations appear in the curves of both the spacing error, velocity, and acceleration, as shown in Figures 8-10, which are obvious in the partially enlarged figures. These fluctuations are reasonable because they are caused by parameter uncertainties, as mentioned above. It is clear that gap errors tend to converge to zero, which is not amplified by the propagation of traffic flow, although uncertainties exist. It proves that the proposed controller (41) renders the UB and UUB performance of the constrained uncertain dynamic CAV platoon system.

\section{Conclusion}

This study proposed a constraint-following control strategy for a CAV platoon system to solve the problem of platoon starting. In these specific processes, the intervehicle distances between consecutive CAVs were considered to construct the control law to guarantee safety and collision avoidance. As the $\mathrm{U}-\mathrm{K}$ approach cannot be directly applied to handle inequality constraints, the diffeomorphism method was adopted to transform the bounded variable into an unbounded variable so that the $\mathrm{U}-\mathrm{K}$ approach could be employed to render the control law. In addition, parametric uncertainties were considered in the designed dynamic system. Thus, an analytical closed-form control law was proposed for this specific dynamic system.

Comprehensive simulations were performed to validate the control performance under the conditions of parametric uncertainty and no parametric uncertainty. The experimental results indicated that the proposed control law rendered by the $\mathrm{U}-\mathrm{K}$ approach could meet the performance requirements of UB and UUB under this specific situation. Moreover, the intervehicle distance was always confined within the specified range, which could guarantee the safety of the CAV platoon system. Meanwhile, there are some limitations to this study. The study was only concerned about the process of platoon starting, however, the stopping also needs to be considered. Furthermore, the acceleration and deceleration of the vehicle platoon can be another key point of platoon control. In the future, we will introduce lateral control into this model to solve the lane-changing and turning problems related to the CAV platoon. Furthermore, we will establish a more sophisticated vehicle dynamic model, including constraints on the spacing error against the current position, measurement noise, communication delay, and mechanical lag, to ensure the safety and stability of the CAV platoon system.

\section{Data Availability}

The data used to support the findings of this study are included within the article.

\section{Conflicts of Interest}

The authors declare that they have no known competing financial interests or personal relationships that could have influenced the work reported in this paper.

\section{Acknowledgments}

This work is supported by National Key Research and Development Program of China (No. 2019YFB1600100), National Natural Science Foundation of China (No. 61973045), Shaanxi Province Key Development Project (No. S2018-YF-ZDGY-0300), Fundamental Research Funds for the Central Universities (No. 300102248403), Joint Laboratory of Internet of Vehicles sponsored by Ministry of Education and China Mobile (No. 213024170015), and 
Application of Basic Research Project for National Ministry of Transport (No. 2015319812060).

\section{References}

[1] D. T. Jia, K. Lu, and J. Wang, "A survey on platoon-based vehicular cyber-physical systems," IEEE Commun. Surv. Tutor.vol. 18, no. 1, pp. 263-284, 2015.

[2] S. E. Li, Y. Zheng, K. Li et al., "Dynamical modeling and distributed control of connected and automated vehicles: challenges and opportunities," IEEE Intelligent Transportation Systems Magazine, vol. 9, no. 3, pp. 46-58, 2017.

[3] E. Moradi-Pari, H. N. Mahjoub, H. Kazemi, Y. P. Fallah, and A. Tahmasbi-Sarvestani, "Utilizing model-based communication and control for cooperative automated vehicle applications," IEEE Transactions on Intelligent Vehicles, vol. 2, no. 1, pp. 38-51, 2017.

[4] B. van Arem, C. J. G. van Driel, and R. Visser, "The impact of cooperative adaptive cruise control on traffic-flow characteristics," IEEE Transactions on Intelligent Transportation Systems, vol. 7, no. 4, pp. 429-436, 2006.

[5] K. C. Dey, L. Yan, X. Wang et al., "A review of communication, driver characteristics, and controls aspects of cooperative adaptive cruise control (CACC)," IEEE Transactions on Intelligent Transportation Systems, vol. 17, no. 2, pp. 491-509, 2016.

[6] M. Wang, W. Daamen, S. P. Hoogendoorn, and B. van Arem, "Cooperative car-following control: distributed algorithm and impact on moving jam features," IEEE Transactions on Intelligent Transportation Systems, vol. 17, no. 5, pp. 1459-1471, 2016.

[7] J. Vanderwelf, S. Shladover, and N. Kourjanskaia, "Modeling effects of driver control assistance systems on traffic," Transp. Res. Rec. J. Transp. Res. Board, vol. 1748, pp. 167-174, 2001.

[8] G. J. L. Naus, R. P. A. Vugts, J. Ploeg, M. J. G. van de Molengraft, and M. Steinbuch, "String-stable cacc design and experimental validation: a frequency-domain approach," IEEE Transactions on Vehicular Technology, vol. 59, no. 9, pp. 4268-4279, 2010.

[9] Q. Xu and R. Sengupta, "Simulation, analysis, and comparison of ACC and CACC in highway merging control," IEEE Intell. Veh. Symp., 2003.

[10] J. Rios-Torres and A. A. Malikopoulos, "A survey on the coordination of connected and automated vehicles at intersections and merging at highway on-ramps," IEEE Transactions on Intelligent Transportation Systems, vol. 18, no. 5, pp. 1066-1077, 2017.

[11] Y. Li, K. Li, T. Zheng, X. Hu, H. Feng, and Y. Li, "Evaluating the performance of vehicular platoon control under different network topologies of initial states," Physica A: Statistical Mechanics and Its Applications, vol. 450, pp. 359-368, 2016.

[12] S. E. Shladover, C. A. Desoer, and J. K. Hedrick, "Automated vehicle control developments in the PATH program," IEEE Transactions on Vehicular Technology, vol. 40, no. 1, pp. 114-130, 2002.

[13] S. E. Shladover, "PATH at 20 - history and major milestones," IEEE Intell. Transp. Syst. Conf., 2006.

[14] Y. Bian, Y. Zheng, W. Ren, S. E. Li, J. Wang, and K. Li, "Reducing time headway for platooning of connected vehicles via V2V communication," Transportation Research Part C: Emerging Technologies, vol. 102, pp. 87-105, 2019.

[15] Z. Wang, Y. Bian, S. E. Shladover, G. Wu, S. E. Li, and M. J. Barth, "A survey on cooperative longitudinal motion control of multiple connected and automated vehicles," IEEE
Intelligent Transportation Systems Magazine, vol. 12, no. 1, pp. 4-24, 2020.

[16] B. Xu, S. E. Li, Y. Bian et al., "Distributed conflict-free cooperation for multiple connected vehicles at unsignalized intersections," Transportation Research Part C: Emerging Technologies, vol. 93, pp. 322-334, 2018.

[17] S. Tak, S. Kim, and H. Yeo, "A study on the traffic predictive cruise control strategy with downstream traffic information," IEEE Transactions on Intelligent Transportation Systems, vol. 17, no. 7, pp. 1932-1943, 2016.

[18] M. Wang, W. Daamen, S. P. Hoogendoorn, and B. van Arem, "Rolling horizon control framework for driver assistance systems. Part I: mathematical formulation and non-cooperative systems," Transportation Research Part C: Emerging Technologies, vol. 40, pp. 271-289, 2014.

[19] Z. Yuan, K. He, and Y. Yang, "A roadway safety sustainable approach: modeling for real-time traffic crash with limited data and its reliability verification," Journal of Advanced Transportation, vol. 2022, Article ID 1570521, 14 pages, 2022.

[20] F. Michaud, P. Lepage, P. Frenette, D. Letourneau, and N. Gaubert, "Coordinated maneuvering of automated vehicles in platoons," IEEE Transactions on Intelligent Transportation Systems, vol. 7, no. 4, pp. 437-447, 2006.

[21] V. Milanes, S. E. Shladover, J. Spring, C. Nowakowski, H. Kawazoe, and M. Nakamura, "Cooperative adaptive cruise control in real traffic situations," IEEE Transactions on Intelligent Transportation Systems, vol. 15, no. 1, pp. 296-305, 2014.

[22] Y. Li, L. Zhang, and H. Zheng, "Nonlane-discipline-based carfollowing model for electric vehicles in transportation-cyberphysical systems," IEEE Transactions on Intelligent Transportation Systems, vol. 19, no. 1, pp. 38-47, 2017.

[23] K. Curts, "Temples and turnpikes in "the world of tomorrow": religious assemblage and automobility at the 1939 New York world's fair," Journal of the American Academy of Religion, vol. 83, no. 3, pp. 722-749, 2015.

[24] J. K. Hedrick, M. Tomizuka, and P. Varaiva, "Control issues in automated highway systems," IEEE Control Systems Magazine, vol. 14, no. 6, pp. 21-32, 1994.

[25] S. S. Stankovic, M. J. Stanojevic, and D. D. Siljak, "Decentralized overlapping control of a platoon of vehicles," IEEE Transactions on Control Systems Technology, vol. 8, no. 5, pp. 816-832, 2000.

[26] G. D. Lee and S. W. Kim, "A longitudinal control system for a platoon of vehicles using a fuzzy-sliding mode algorithm," Mechatronics, vol. 12, no. 1, pp. 97-118, 2002.

[27] G. Antonelli and S. Chiaverini, "Kinematic control of platoons of autonomous vehicles," IEEE Transactions on Robotics, vol. 22, no. 6, pp. 1285-1292, 2006.

[28] A. Ghasemi, R. Kazemi, and S. Azadi, "Stable decentralized control of a platoon of vehicles with heterogeneous information feedback," IEEE Transactions on Vehicular Technology, vol. 62, no. 9, pp. 4299-4308, 2013.

[29] J. I. Ge and G. Orosz, "Optimal control of connected vehicle systems with communication delay and driver reaction time," IEEE Transactions on Intelligent Transportation Systems, vol. 18, no. 8, pp. 2056-2070, 2017.

[30] N. Chen, M. Wang, T. Alkim, and B. van Arem, "A robust longitudinal control strategy of platoons under model uncertainties and time delays," Journal of Advanced Transportation, vol. 2018, no. 2, pp. 1-13. In press, 2018.

[31] F. Dong, X. Zhao, J. Han, and Y. H. Chen, "Optimal fuzzy adaptive control for uncertain flexible joint manipulator based 
on D -operation," IET Control Theory \& Applications, vol. 12, no. 9, pp. 1286-1298, 2018.

[32] Z. Yan, M. Wang, and J. Xu, "Global adaptive neural network control of underactuated autonomous underwater vehicles with parametric modeling uncertainty," Asian Journal of Control, vol. 21, no. 3, pp. 1342-1354, 2018.

[33] G. D. Lee and S. W. Kim, "A longitudinal control system for a platoon of vehicles using a fuzzy-sliding mode algorithm," Mechatronics, vol. 12, no. 1, pp. 97-118, 2002.

[34] C. Desjardins and B. Chaib-Draa, "Cooperative adaptive cruise control: a reinforcement learning approach," IEEE Transactions on Intelligent Transportation Systems, vol. 12, no. 4, pp. 1248-1260, 2011.

[35] E. Kayacan, "Multiobjective $\$ \mathrm{H}_{-}\{$linfty $\}$\$ control for string stability of cooperative adaptive cruise control systems," IEEE Transactions on Intelligent Vehicles, vol. 2, no. 1, pp. 52-61, 2017.

[36] S. Wen, G. Guo, and X. Su, "Cooperative control of vehicle platoons according to driving visibility status," Control Theory \& Applications, vol. 36, no. 7, pp. 1153-1164, 2019.

[37] F. E. Udwadia and R. E. Kalaba, Analytical Dynamics. A New Approach, Cambridge University Press, 1996.

[38] C. M. Pappalardo, "A natural absolute coordinate formulation for the kinematic and dynamic analysis of rigid multibody systems," Nonlinear Dynamics, vol. 81, no. 4, pp. 1841-1869, 2015.

[39] X. Liu, S. Zhen, and K. Huang, "A systematic approach for designing analytical dynamics and servo control of constrained mechanical systems," IEEE/CAA Journal of Automatica Sinica, vol. 002, no. 004, pp. 382-393, 2015.

[40] R. Yu, Y.-H. Chen, H. Zhao, K. Huang, and S. Zhen, "Selfadjusting leakage type adaptive robust control design for uncertain systems with unknown bound," Mechanical Systems and Signal Processing, vol. 116, pp. 173-193, 2019.

[41] Z. Hu, Z. Yang, J. Huang, and Z. Zhong, "Safety guaranteed longitudinal motion control for connected and autonomous vehicles in a lane-changing scenario," IET Intelligent Transport Systems, vol. 15, no. 2, pp. 344-358, 2021.

[42] H. Yin, Y.-H. Chen, and D. Yu, "Vehicle motion control under equality and inequality constraints: a diffeomorphism approach," Nonlinear Dynamics, vol. 95, no. 1, pp. 175-194, 2019.

[43] F. E. Udwadia and R. E. Kalaba, "On the foundations of analytical dynamics," International Journal of Non-linear Mechanics, vol. 37, no. 6, pp. 1079-1090, 2002.

[44] Y.-H. Chen, "Constraint-following servo control design for mechanical systems," Journal of Vibration and Control, vol. 15, no. 3, pp. 369-389, 2009.

[45] B. Noble and J. W. Daniel, "Applied linear algebra," Mathematical Gazette, vol. 72, no. 462, 1988.

[46] Y.-H. Chen and X. Zhang, "Adaptive robust approximate constraint-following control for mechanical systems," Journal of the Franklin Institute, vol. 347, no. 1, pp. 69-86, 2010.

[47] R. Zou, J. Sun, and J. Sun, "Leader-following constrained distributed adaptive dynamic programming design for multiagent systems," Chinese Automation Congress (CAC), pp. 5345-5349, 2019. 